\title{
Hígado graso agudo en el embarazo
}

\author{
Acutte fatty liver of pregnancy
}



'Dra. Natalia Villegas Loaiza

Poder Judicial, San José, Costa Rica navilo 88@hotmail.com

https://orcid.org/0000-0001-7639-8324

$\begin{array}{lcc}\text { RECIBIDO } & \text { CORREGIDO } & \text { ACEPTADO } \\ 09 / 03 / 2019 & 15 / 03 / 2019 & 23 / 03 / 2019\end{array}$

\section{RESUMEN}

El hígado graso agudo en el embarazo, conocido en sus siglas en inglés como AFLP (Acutte Fatty Liver of Pregnancy), es una complicación grave muy poco común y exclusiva del embarazo que conlleva a una insuficiencia hepática aguda y potencialmente fatal. Dicha patología amerita un diagnóstico y tratamiento diligente con el propósito de evitar aumentos en la morbimortalidad materna y fetal, y se relaciona con la conclusión del embarazo para su cese.

PALABRAS CLAVE: complicaciones del embarazo; fallo hepático; hígado graso.

\section{ABSTRACT}

Acute Fatty Liver in Pregnancy is a serious and very rare complication

${ }^{1}$ Médico general, graduada de exclusive of pregnancy which leads to acute and potentially fatal liver la Universidad de Iberoamérica (UNIBE), médico de empresa en Poder Judicial, San José, Costa Rica. Código médico: 12040. failure. AFLP is a pathology that requires a diagnosis and diligent treatment in order to avoid maternal and fetal morbidity and mortality, and it is related to the end of pregnancy for its cessation.

KEYWORDS: pregnancy complications; liver failure; fatty liver.

\section{INTRODUCCIÓN}

El hígado graso agudo es una enfermedad exclusiva del embarazo, la cual se caracteriza por la aparición repentina de una insuficiencia hepática (8) que se desarrolla durante el tercer trimestre, aunque no es exclusiva de este (10), ya que existen reportes de casos que se presentan a las 23 semanas de edad gestacional (10). Sin embargo, es común que esta enfermedad aparezca a la semana 36 de gestación, con un rango entre las 32 y 38 semanas. Esta emergencia obstétrica alcanzaba altas tasas de mortalidad materno-fetales que han disminuido sustancialmente (10) 
debido a las mejoras en su diagnóstico y pronta respuesta terapéutica. Su evolución puede ser o no aguda, se caracteriza por ser inespecífica (10) y asocia una sintomatología variable (13).

Es importante conocer y comprender esta enfermedad, ya que es poco frecuente y expone una patología grave, por lo que el médico debe sospecharla para no pasarla por alto y realizar un diagnóstico precoz. Este artículo de revisión tiene como propósito, describir la patología, su presentación clínica, diagnóstico y abordaje terapéutico, enfatizado a que éste último es particularmente importante para disminuir el alto riesgo al que se exponen la madre y su hijo; además, esta alteración se puede relacionar con la afectación a otros órganos como por ejemplo el riñón, páncreas, entre otros (1).

El objetivo de éste análisis, también es ilustrar al lector sobre la importancia de considerar el hígado graso agudo dentro del diagnóstico diferencial, al igual que otras hepatopatías graves en las que la labor médica final es concluir el embarazo llevando a cabo el parto, tomando en cuenta que no hay hasta el momento, un tratamiento específico.

\section{EPIDEMIOLOGÍA}

Fue descrita por primera vez en el año 1934 por Stander y Cadden, investigadores que la bautizaron como una "atrofia amarilla aguda del hígado" (7), y se caracteriza porque produce una infiltración grasa de los hepatocitos maternos provocando un proceso inflamatorio agudo y severo que conlleva a un fallo hepático, rara vez se manifiesta durante el post parto, pero si existen casos señalados (13) Asimismo, se presenta en 1 de 1000 embarazos (15). Además, no se conoce con exactitud cuál es su causa, pero se ha relacionado con una disfunción mitocondrial que desata una perturbación en la betaoxidación de ácidos grasos, desatando una interacción materno - fetal que transige la enfermedad.

\section{ETIOPATOGENIA}

Fisiológicamente, durante la actividad o en periodos de ayuno los ácidos grasos son moléculas que se oxidan para que se conviertan en fuentes de energía para el cerebro, corazón y músculo esquelético. En AFLP existe una deficiencia de la enzima 3 hidroxiacil-CoA deshidrogenasa de cadena larga (LCHAD) producido por una mutación Autosómica recesiva (6) debido a una variación del gen ACADM que encausa un exceso de ácidos grasos de cadena larga a nivel fetal y llegan a la circulación materna y sobrepasan la competencia de la función hepática de la madre y provocan un fallo hepático (7).

\section{FACTORES DE RIESGO}

El desarrollo de esta patología se ha asociado a diversos factores de riesgo, tales como: embarazos múltiples, el uso prolongado del ácido acetil salicílico, edad materna avanzada, producto masculino, obesidad y madres primigestas $(2,5)$. Sin embargo, a pesar de los múltiples factores de riesgo vinculados al desarrollo de la enfermedad, no hay factores predictores que se manifiesten durante el transcurso de la gestación para prevenir el progreso del trastorno. 


\section{MANIFESTACIONES CLÍNICAS}

Los síntomas iniciales suelen ser inespecíficos y progresivos (2), incluidos desde malestar general, náuseas, vómitos, dolor abdominal, epigastralgia, síntomas de hipoglucemia, ascitis ictericia, fiebre hasta encefalopatía (2). También se ha reportado la presencia de lesiones en la piel tipo maculo-papular inespecífica sin prurito que suele ser auto limitada (13) y hasta en el $50 \%$ de las pacientes se detecta edema, proteinuria e hipertensión arterial (2)

\section{DIAGNÓSTICO}

El Gold Estándar para el diagnóstico es la biopsia hepática (2), la cual evidencia la esteatosis microvascular; pero no es común realizarla en los centros hospitalarios por la necesidad de un diagnóstico oportuno y un manejo precoz, por lo que se usa un conjunto de criterios clínicos y de laboratorio para el diagnóstico (11).

Histológicamente, se observa infiltración grasa microvesicular de los hepatocitos con signos de inflamación. Asimismo, se observa con microscopía electrónica alteraciones en el tamaño y forma mitocondrial (1).

Los Criterios de Swansea ayudan a simplificar el trabajo diagnóstico en caso de sospecha clínica (TABLA 1) y se necesitan entre 6 o más criterios presentes para el diagnóstico (13). Estos criterios van desde los síntomas inespecíficos antes descritos hasta cambios bioquímicos en los exámenes de laboratorio que incluyen: hipoglicemia, aumento de leucocitos, bilirrubina, amoniaco sérico, creatinina y de transaminasas, hasta alteraciones de las pruebas de coagulación con prolongación de la protrombina y la disminución del fibrinógeno.

Los estudios de imágenes han demostrado tener baja sensibilidad, siendo el ultrasonido la modalidad con mayor valor que la tomografía axial computarizada (TAC) $(1,7)$ aunque no se recomiendan para definir el diagnóstico.

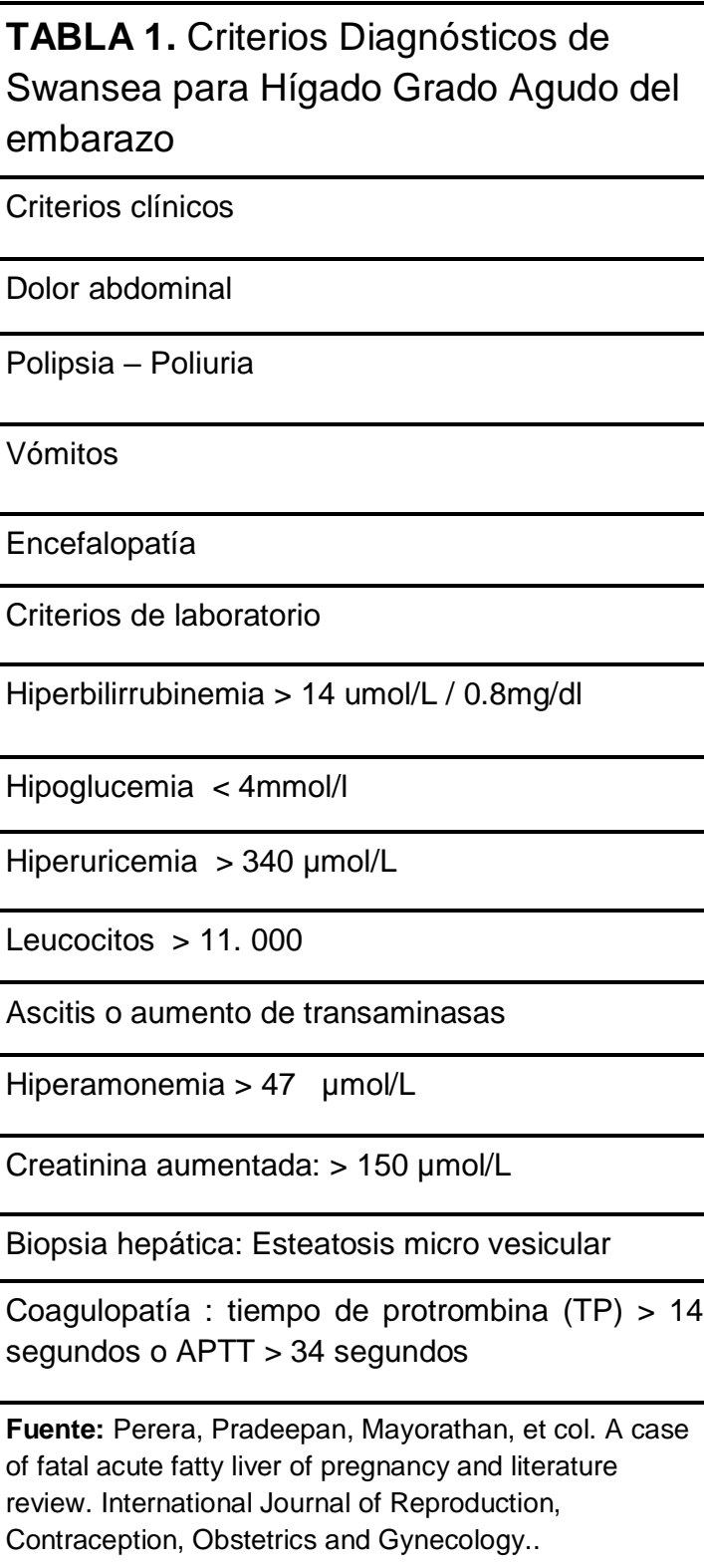




\section{DIAGNÓSTICO DIFERENCIAL}

El diagnóstico diferencial comprende desde Síndrome de HELLP, hepatitis virales 0 autoinmunes hasta fallo hepático inducido por drogas (7). La inespecificidad del cuadro clínico puede sobrellevar a errores diagnósticos y a confundir el cuadro con otras patologías hepáticas.

\section{COMPLICACIONES}

A principios del Siglo $X X$, se reportaban tasas de mortalidad materna y fetal de hasta el $80 \%$ (8). Actualmente, esta patología asocia un $10 \%$ de mortalidad y complicaciones maternas, y un $23 \%$ fetales, en su mayoría de casos a consecuencia de la prematuridad (15), gracias a un diagnóstico más precoz en la actualidad.

Esta patología se presenta como una emergencia obstétrica reversible con el parto, que puede llegar a afectar otros órganos concomitantemente en casos de cuadros graves, como alteración de la creatinina y fallo renal agudo (9), siendo usualmente una alteración renal moderado sin ameritar diálisis (9). Además, puede presentar trombocitopenia, coagulación intra vascular diseminada (CID), encefalopatía hepática por aumento de amonio, hemólisis, sepsis y la pancreatitis que llega a ser una complicación letal. En algunas pacientes se requiere trasplante hepático, no obstante, es ocasional (10).

\section{TRATAMIENTO}

El tratamiento inicial más importante es la estabilización de la madre, mediante el cual debe corregirse la hipoglicemia con
Dextrosa al 5\% manteniendo los niveles de glucosa estable, manejo del control de líquidos conservando una balance de la función renal, además de tener un control sobre el alto riesgo de sangrado que tiene la paciente, monitorizando las pruebas de coagulación, niveles de plaquetas, hemoglobina y corregir las alteraciones encontradas, valorando constantemente el estado mental de la paciente.

Dado que, se trata de una enfermedad que es reversible con el parto, y que la circunstancia patológica del hígado suele ser auto limitada (13), por lo que la interrupción del embarazo provocando el parto, se convierte en el tratamiento definitivo.

La vía de parto dependerá de la individualización de cada caso, en vista del compromiso materno y fetal. Aunque se describe que el parto vaginal continúa siendo la opción más viable, algunas madres ameritan parto por cesárea en caso de existir un deterioro de la condición clínica de alguno de los dos pacientes (3).

El uso de plasmaféresis posterior a la interrupción del embarazo se emplea en el manejo de casos avanzados o severos de la enfermedad asociado a mayores complicaciones. Este tratamiento es basado en remover las sustancias tóxicas como amonio, bilirrubinas y citokinas inflamatorias de la circulación hepática, reemplazando el plasma de la paciente (12), con el objetivo de mejorar la función hepática y el daño renal. Asimismo, ha revelado ser eficaz en pacientes en espera de trasplante hepático como implementación prematura cuando coexisten agravantes que amenacen la vida. La plasmaféresis mejora los síntomas, reduce la estancia 
hospitalaria y detiene la progresión de la enfermedad (6).Los casos de recurrencia de hígado graso agudo pueden presentarse en embarazos posteriores hasta en un $25 \%$ de los casos (3), ya que el fallo hepático es desencadenado por la interacción materno fetal, por lo que se recomienda consejería genética en el post parto (2) con análisis en el recién nacido y el estudio de la mutación del gen HDAHA para el control de futuros embarazos. Además, se ha sugerido un monitoreo del neonato, debido a que algunos han relacionado la presencia de la enfermedad hepática materna, con la aparición de deficiencia de vitamina $\mathrm{K}$ en el niño y con ello se busca una reducción de morbilidad y mortalidad del neonato por complicaciones secundarias a la deficiencia vitamínica (6).

\section{CONCLUSIONES}

Debido a que el hígado graso agudo en el embarazo es poco común, debemos sospecharlo para realizar un diagnóstico temprano por las complicaciones que conlleva, obteniendo un manejo oportuno con el fin de disminuir la morbimortalidad materno-fetal.

Esta patología requiere de integrar un adecuado soporte a la madre y al feto, así como la inducción de la labor de parto inmediato y el posterior monitoreo tanto de la madre como del recién nacido por posibles complicaciones que se desarrollen en el post parto, especialmente, si existe el antecedente de la enfermedad en embarazos previos. Finalmente, a pesar de que la biopsia es el estándar de oro para el diagnóstico, por su limitada accesibilidad, se puede basar el diagnóstico en los Criterios de Sweansa que facilitan el trabajo al clínico cuando existe la sospecha diagnóstica. Así que, debido a la gravedad de dicha enfermedad es importante conocerla para efectuar un adecuado abordaje.

\section{REFERENCIAS}

1. Han XD, Cao X, Gu GF. Analysis of seven consecutive cases of acute fatty liver of pregnancy: single center experience in China. Int J Clin Exp Med. 2017;10(9): 3938-13943.Disponible en: http://www.ijcem.com/files/ijcem0057775.pdf

2. Duarte Mote J, Díaz Meza S, Lee Eng Castro V, Castro Bravo J, Velásquez Díaz V. Hígado graso agudo del embarazo: experiencia de 8 años. Medicina Interna de México. 2007;23(5): 464-70. Disponible en: http://www.medigraphic.com/pdfs/medintmex/mim-2007/mim0750.pdf

3. Al-Husban N, Al-Kuran O, Al Helou A. Postpartum acute fatty liver of pregnancy: a case report. Journal of Medical Case Reports. 201806 01;12(67):1-6. https://doi.org/10.1186/s13256-018-1593-3

4. Sepulveda-Martinez A, Romero C, Juárez G, Hasbun J, Parra-Cordero M. Actualización en el diagnóstico y manejo del daño hepático agudo grave en el embarazo. Revista Médica Chile. 2015;143:627-636. Disponible en: https://scielo.conicyt.cl/pdf/rmc/v143n5/art11.pdf

5. Perera IA, Pradeepan JA, Mayorathan U, Kumanan Thirunavukarasu Kum T, Muhunthan K, Selvaratnam G. A case of fatal acute fatty liver of pregnancy and literature review. International Journal of 
Reproduction, Contraception, Obstetrics and Gynecology. $2018 \quad 04$ 28;7(5):2026-2030. https://doi.org/10.18203/2320- 1770.ijrcog201819507

6. Klauser C, Saltzman D. Enfermedades gastrointestinales en el embarazo. Diagnóstico y tratamiento Gineco obstetricos. XI Edición, Mexico: McGraw-Hill Education; 2014: 493-500.

7. Gorginzadeh M, Safari S, Alavian SM. Acute Fatty Liver of Pregnancy: A Life-Threatening Condition Requiring a Life-Saving Approach. Hepatitis Monthly. 201605 25;16(6): e35256 . https://doi.org/10.5812/hepatmon.35256

8. Cano F, Penna M, Aedo S. Hígado graso agudo del embarazo, reporte de dos casos clínicos y revisión de la literatura. Revista Obstet Ginecol - Hospital Santiago Oriente Dr Luis Tisné Brousse. 2012;7(1):34-38. Disponible

en: https://www.researchgate.net/publication/251573815 Higado graso agudo del embarazo reporte de d os casos clinicos y revision de la literatura

9. Gao Q, Qu X, Chen X, Zhang J, Liu F, Tian S, Wang C. Outcomes and risk factors of patients with acute fatty liver of pregnancy: a multicentre retrospective study. Singapore Medical Journal. 2018 08;59(8):425430. https://doi.org/10.11622/smedj.2018001

10. Westbrook RH, Dusheiko G, Williamson C. Pregnancy and liver disease. Journal of Hepatology. 2016 04;64(4):933-945. https://doi.org/10.1016/i.jhep.2015.11.030

11. Santana L, Hernández Medina E, O\&\#39;Shanahan G, Sánchez-Palacios M. Fallo renal agudo en el hígado graso agudo del embarazo: a propósito de un caso. Revista de nefrología. 2005;25(4): 453-454. Disponible en: http://www.revistanefrologia.com/es-pdf-X021169950501849

12. Trang HNK, Tuyet HTD. Case Report: Acute Fatty Liver of Pregnancy. Open Journal of Obstetrics and Gynecology. 2017;07(10):1017-1023. https://doi.org/10.4236/ojog.2017.710102

13. Seyyed Majidi MR, Vafaeimanesh J. Plasmapheresis in Acute Fatty Liver of Pregnancy: An Effective Treatment. Case Reports in Obstetrics and Gynecology. 2013;2013:1-5. https://doi.org/10.1155/2013/615975

14. Rebahi H, Still ME, El Adib AR. A successful use of therapeutic plasma exchange in a fulminant form of acute fatty liver of pregnancy. Journal of Gynecology Obstetrics and Human Reproduction. 2019 02;48(2):133-137. https://doi.org/10.1016/i.jogoh.2018.10.001

15. Gabbe S. Obstetrics: Normal and Problem Pregnancies. VI Edición. Philadelphia: Elsevier; 2017. 